\title{
Experiencia de articulación del discurso de la memoria en un taller de relatos del terremoto y tsunami de 1960
}

\author{
Experience of Articulation of Memory Discourse in a Workshop \\ on Stories of the Earthquake and Tsunami of 1960 \\ Experiência de articulação do discurso da memória em uma \\ oficina de relatos do terremoto e tsunami de 1960
}

\section{Adriana Huaiquimil Hermosilla ${ }^{1}$ Cristian Abarca González ${ }^{2}$}

\section{Resumen}

Este artículo describe la experiencia de reconstrucción de la memoria en torno al terremoto y tsunami de 1960, por parte de un grupo de sobrevivientes a este evento catastrófico. Para este estudio se ha utilizado metodología cualitativa, la que ha incluido la narración como ejercicio escritural, además de entrevistas en profundidad. Se efectuaron 10 talleres de narrativa y conversatorios con un grupo de 15 personas de Maullín, territorio costero del sur de Chile. Del análisis de los resultados se advierte que las representaciones de la memoria del evento de los miembros de la comunidad siguen patrones semánticos y simbólicos anclados en la tradición cristiana, la familia y la construcción de la identidad en torno al territorio. La ideación de tales formas constituye los marcos sociales que conforman la articulación de la narración testimonial.

\section{Palabras clave}

memoria; narración; marco social; terremoto

\section{Abstract}

This article describes the memory reconstruction experience around the 1960 earthquake and tsunami by a group of survivors to this catastrophic event. For this study, we have used a qualitative method, including narrative as a scriptural exercise, as well as in-depth interviews. Ten narrative workshops and talks were held with a group of 15 people from Maullín, a coastal territory in southern Chile. Based on the analysis of the results, we see that the representations of the memory of the event of the members of the community follow semantic and symbolic patterns anchored in the Christian tradition, the family and the construction of the identity around the territory. The ideation of such forms constitutes the social frameworks that make up the articulation of testimonial narration.

\section{Keywords}

memory; narration; social framework; earthquake

\section{Resumo}

Este artigo descreve a experiência de reconstrução da memória em torno ao terremoto e tsunami de 1960, por parte de um grupo de supervivientes a este sucesso catastrófico Para este estudo utilizou-se metodologia quantitativa, que inclui a narração como exercício de escrita, além de entrevistas em profundidade. Efetuaram-se 10 oficinas de narrativa e diálogos com um grupo de 15 pessoas de Maullín, território na costa do sul do Chile. A partir da análise dos resultados, evidencia-se que as representações da memória do evento dos membros da comunidade seguem padrões semânticos e simbólicos fiéis à tradição cristã, à família e à construção da identidade em torno ao território. A ideação dessas formas constitui as estruturas sociais que compõem a articulação da narração testemunhal.

\section{Palavras-chave}

memória; narração; estrutura social; terremoto

Artículo recibido el 10 de abril de 2017 y aprobado el 10 de enero de 2018

1 Universidad San Sebastián, Puerto Montt, Chile. Correo electrónico: adriana.huaiquimil@uss.cl

2 Liceo de Adultos Francisco Tello González, Rancagua, Chile. Correo electrónico: cabarcagonzalez@hotmail.com 


\section{Introducción}

El 22 de mayo de 1960 pasadas las tres de la tarde, el pueblo de Maullín, al igual que otros territorios del centro sur de Chile, fue azotado por el megaterremoto y posterior tsunami ${ }^{3}$ registrado como el más grande ocurrido en la historia moderna de la humanidad. Este pueblo costero fue devastado principalmente por la llegada de ocho grandes olas al estuario del río Maullín, de las cuales la segunda y la cuarta fueron las más altas registradas con una altura máxima de 14 metros (Atwater et al., 2013).

La participación testimonial sobre esta catástrofe ha recaído más fuertemente sobre la devastación de grandes ciudades, relegando a un segundo plano a los habitantes de territorios rurales y más apartados. En sectores como el caso que se analiza, el rescate y difusión de relatos testimoniales entre las generaciones presentes y futuras es una forma de fortalecer la comunidad, otorgar protagonismo a sus voces $y$ fortalecer su participación en la construcción de la memoria.

Desde el ejercicio de la memoria como práctica textual y social se exaltan herramientas y conocimientos compartidos culturalmente para afrontar futuras amenazas socionaturales ${ }^{4}$, los que cobran aun mayor importancia dados los resientes movimientos telúricos acaecidos en el país, como el terremoto y posterior tsunami de $2010 \mathrm{o}$ el reciente terremoto de 2015 que azotó a la zona centro norte del país.

En este sentido, los relatos testimoniales ofrecen un bagaje de conocimiento sociocultural que bajo códigos simbólicos y referenciales se desprenden para reconstruir episodios de vida asociados al cataclismo y a aprendizajes comunitarios para la

3 Tuvo su epicentro telúrico en la ciudad de Valdivia, mientras el tsunami se desplegó por gran parte de la costa chilena con una magnitud estimada en Mw 9,5, causando más de 2000 muertes en el país, 61 personas en Hawái y 122 en Japón, debido a su propagación al Pacífico (Atwater et al., 2013; Santillán, Keller y Cisterna, 2010; Schuster, Salcedo y Valenzuela, 2002).

4 Nos referimos a este tipo de eventos como desastre socionatural considerando que en la ocurrencia del impacto y el posterior proceso inciden factores ambientales, sociales y psicológicos que permiten abodar desde una mirada compleja la forma en que las comunidades son mayor o menormente afectadas más allá de la perspectiva física (Maskrey, 1993). reconstrucción y reterritorialización. Si bien en el relato testimonial se avizoraban lazos con la cronología de la historia del acontecimiento, no se pretende conocer la realidad de los hechos ocurridos en el pasado, sino penetrar en las representaciones en torno a ese pasado (Beverley y Achugar, 1992; Randall, 1992). "No se trata de convertir las narraciones en objetos de conocimiento [...] para acercarnos a una verdad sobre la realidad indagada. Contrario a ello, el objetivo es reconocer el potencial transformador de la narración y del lenguaje" (Pinilla, 2011, p. 20).

La experiencia de los asuntos humanos adquiere la forma de narraciones que se utilizan para contar cosas sobre ellos. Así, es posible acceder al sentido y construcción del discurso de los eventos mediante el relato y, a su vez, el discurso permite la resignificación de acontecimientos como reconstrucción de un pasado vivido que se contiene en marcos sociales y en significados alojados en la cultura (Halbwachs, 2004). De este modo, los acontecimientos y la experiencia se enmarcan en formas narradas que actúan como marcos de la memoria y proporcionan un contexto interpretativo al mismo tiempo.

La narración siempre ha dado cuenta de los desafíos, las esperanzas, los horrores y de la vida común de las personas. La manera en que una comunidad se textualiza no se termina a menos que los acontecimientos caigan en el olvido. Sin embargo, este puede terminarse con las luces de la memoria y, así, traer y recrear las situaciones acaecidas en el pasado (Nora, 2001; Ricoeur, 1999). El ejercicio/acto de la memoria narrativa implica una constante traslación del pasado a un presente enunciativo y con ello su reactualización con miras a un devenir social de progreso para todos los sujetos involucrados en la vida comunitaria (Corti, Moreno y Widow, 2011; Ricoeur, 1983).

Desde tal aproximación como punto de inicio, el interés de esta investigación se centró en conocer mediante la praxis de la narración el proceso de construcción, deconstrucción y reconstrucción de las memorias del evento en la producción del discurso narrativo de los supervivientes de la catástrofe 
de 1960 en la comuna de Maullín, región de Los Lagos, sur de Chile.

Para ello se propuso realizar un taller de narrativa y conversatorio con adultos mayores de la localidad La Pasada, quienes vivenciaron y sobrevivieron a la catástrofe de 1960. El estudio se enfocó en el caso de esta comunidad dada su invisibilización, entre otros ámbitos, discursiva en el contexto testimonial del evento. El taller tuvo por objetivo traspasar técnicas básicas narrativas y promover espacios de encuentro comunitario para impulsar la producción del discurso narrativo de los supervivientes en torno al evento catastrófico desde sus propias significaciones.

Entendiendo los marcos sociales como ejes articuladores del discurso de la memoria, nos preguntamos: ¿Cuáles son los significados culturales que operan como marcos sociales en los relatos que componen el corpus?; ¿cómo estos significados culturales articulan la resignificación de las representaciones simbólicas de las vivencias del evento experimentadas por este grupo de sobrevivientes?

El supuesto que guía este trabajo es que podemos acceder a la construcción, deconstrucción y reconstrucción de la memoria en la producción del discurso narrativo de la comunidad estudiada mediante la interpretación de los patrones simbólicos y culturales que operan en ella como marcos sociales que articulan la narración, estos patrones son los que le dan una forma textual específica a la memoria.

El texto se organiza en las siguientes secciones. Se presenta, en primer lugar, una discusión teórica en torno a la textualización del evento como ejercicio escritural y como constructo cultural y social a partir de la evolución genérica de la narración del evento y su función como correlato de hechos acontecidos en la realidad. Luego se expone la experiencia del taller de narrativa como estrategia de emergencia de la memoria y posteriormente se develan los principales hallazgos del trabajo referidos, en primer lugar, a los marcos sociales que operan en los relatos y, a continuación, la descripción de la experiencia por parte de los sujetos participantes de la intervención. Se concluye con algunas reflexiones en torno a la construcción activa e intersubjetiva de las memorias alrededor del evento catastrófico.

\section{Marco teórico}

El tejido de la memoria implica un ejercicio narrativo por parte del (de los) sujeto(s) que busca(n) establecer una discursividad a partir del recuerdo de hechos y eventos. Los recuerdos, así como el ejercicio de la memoria, se dan a partir de la confrontación con otros sujetos, incluso de uno mismo como sujeto desdoblado. Por tal motivo, el ejercicio de la memoria se ha definido como una praxis social y textual, punto de partida de la revisión teórica que se ofrece a continuación. En el segundo apartado se aborda la narración como realidad o ficción, según Bajtín (1999), Presas (1997), y Martínez y Scheffel (2011), para terminar con la partición textual en lexías y códigos otorgadores de sentido propuesta por Barthes (2004). De estos últimos se recurre al código semántico y al código simbólico para realizar los análisis del corpus de relatos de este trabajo a la luz de los marcos que articulan el discurso de la memoria.

\section{La memoria como ejercicio social}

y textual: el relato de la comunidad

La arquitectura de la memoria y el acceso a ella se realizan desde la narración, los sujetos memorizantes hacen una reconstrucción de la realidad desde esta forma textual, lo que implica que la realidad es construida narrada y, al mismo tiempo, es narración: la textualidad narrativa.

Esta relación que ha marcado al ser humano desde los albores de la cultura y de su formación como sujeto capaz de dar cuenta de sí mismo ${ }^{5}$, se manifiesta en el mito como fundamento de las relaciones sobrenaturales y sagradas, es decir, se erige desde la narración. Desde allí se puede señalar que las comunidades actuales textualizan neomitos y micromitos como constructo cultural y social que

5 Dar cuenta de sí mismo implica un ejercicio de separación del contexto inmediato para reflexionar sobre él y devenirlo discurso, situación que evidencia cierta capacidad de abstracción del (de los) sujeto(s) que realiza(n) la acción narrativa. 
oficia de correlato discursivo de los hechos acontecidos en la realidad. En definitiva, toda comunidad requiere de ciertos textos que den cuenta de lo que es, ha sido y quiere ser, así como una nación se cimienta en ideas-fuerza que orientan su identidad narrativa y mítica (Subercaseaux, 2004; Jelin, 2002; Candau, 2008). Lo mismo ocurre en una comunidad desde la visión particular que se perfila en la acción de sus sujetos y el territorio que ocupan (Chávez, 2007). Por lo que este ejercicio puede ser de carácter sagrado (ya sea como origen, final, explicación de ciertos eventos, etc.) y político (como manera de relación inter- e intracomunidades), dependiendo de la colectividad que textualiza y de los fines con que los grupos dirigentes y de influencia pretenden orientar tales relatos, por tanto, el territorio es un constructo geopolítico y simbólico.

Por otra parte, la relación de la realidad como construcción narrativa y la narración como constructo de la realidad es dialéctica, pues el ejercicio de la narración otorga sentido y ordenamiento al mundo, al tiempo que el mundo, desde los patrones socioculturales, entrega un marco que funciona como articulación de la narración. Esto quiere decir que ambas esferas están en simbiosis constante: la realidad textualizada y la textualización de la realidad.

Los relatos que dan cuenta de una comunidad serán montados desde cierta temporalidad y espacialidad, aportando a este binomio conceptual un marco de referencia que es el marco narrativo o marco social (Mendoza, 2004). "Cuando las personas hacen memoria, lo que hacen es interpretar y construir la realidad de acuerdo con la socialización y el sistema normativo con que los individuos han guiado y guían sus vidas" (Lynch y Oddone, 2008, p. 130).

Ante este escenario, la memoria selecciona y recrea aquello que es significativo para el cometido de la comunidad, es decir, para los valores que quiere destacar o marcar como carta de navegación de su devenir ${ }^{6}$ la memoria es un ejercicio de narración

6 Esto implica también destacar antivalores con su respectivo castigo, muchas veces la narración de la memoria de una comunidad sigue patrones de fábula con un sentido didáctico para los futuros sujetos de la comunidad. Al respecto, Bruner (1990) señala que lo que no se narra se pierde, se entiende, entonces, que los valores y antivalores afectivo; lo que no sirve es desechado, olvidado, invisibilizado u ocultado. En esta relación se imbrica también lo sagrado y lo político como simbología social particular.

El mencionado marco social asegura la fijeza $y$ coherencia de los recuerdos (Mendoza, 2004, p. 4) además de darle corporeidad al discurso de la memoria, trabajando como un tipógrafo de esta plasmación discursiva. El suceso que se recuerda y se hace narración a través del paso del marco social debe cumplir el requisito de ser un suceso memorable, tal exigencia se encuentra en las concepciones de la comunidad. En este sentido, los acontecimientos traumáticos son un material muy moldeable para el discurso de la memoria, dado que ponen a prueba la capacidad de reacción y de sobrevivencia de los sujetos, así como de las entidades sociales que operan desde lo afectivo y político.

El marco social cumple la función de un dispositivo de mediación entre lo ocurrido y la forma en que se textualizará, dentro de este marco se traman patrones simbólicos y culturales que se aprecian como la filiación a cierta tradición moral y religiosa con que la comunidad discurseante busca darle verosimilitud a sus relatos y al proyecto que pretende sustentar con la conformación de estos.

La memoria que se teje en los relatos testimoniales no es un corpus cerrado y que no admite re-escrituras ${ }^{7}$; por el contrario, al tratarse de historias comunes a un grupo de personas está sujeta a interpretaciones de los mismos sujetos que motiva el devenir del espacio y tiempo (Bruner, 1990). Por consiguiente, el territorio funciona como un universo semiótico que genera constantes imbricaciones de la retórica memorialística, los sujetos construyen relatos que devienen desde informar, entender, apropiar, alegorizar, mitificar, etc., que van fluctuando entre las aproximaciones cognitivas y discursivas

seleccionados en la textualización tienen la misión de construir modelos y antimodelos funcionales a las pretensiones sociales y comunitarias.

7 Es el texto quebrado del que habla Barthes en S/Z (2004), aquel que se puede relacionar con otros textos y generar nuevos, enriquecerse, cambiar, devenir, etc. La particularidad que tiene es que la relación con otras textualidades le otorga la potencialidad de constante cambio: texto y memoria mutables. 
(Mendoza, 2004, p. 3) con la finalidad de dar cuenta de un evento o situación, la primera, mientras que la segunda aproximación se orienta hacia la circulación de los relatos y su intertextualización, generando una matriz que textualiza desde un relato anterior y que posee la carga de otras memorias, es decir, de elementos culturales palimpsésticos.

De esta manera, los sujetos van reconfigurando su relato y generando un metarrelato que constituye su identidad y termina por organizar un nuevo marco social desde el que se textualizarán nuevos acontecimientos venideros o acontecimientos acallados en el pasado y que se quieren visibilizar.

\section{Narración: realidad y ficción}

El ejercicio de la memoria y los marcos sociales se plantean a partir de la narración, situación que se valida desde lo siguiente: "1. Es posible narrar procesos reales o inventados. 2. Es posible narrar en el marco del discurso cotidiano o en el marco del discurso poético" (Martínez y Scheffel, 2011, p. 20). Esto quiere decir que la narración se puede plantear con un correlato en la realidad de la comunidad emplazada (como es el caso del taller) y ficcionalizarla sin que se desconozca del todo el mencionado correlato o empleándolo como punto de anclaje de la textualidad que se está textualizando.

Frente a esta situación de articulación entre realidad y ficción se puede hablar de un género discursivo primario y un género discursivo secundario, así como de la transición entre ambos (Bajtín, 1999, p. 289), pues la socialización oral de ciertos eventos se ha textualizado conformando un discurso narrativo de un acontecimiento vivido y repetido ${ }^{8}$. Al respecto, Bajtín señala que los géneros discursivos primarios corresponden a formas empleadas en la vida cotidiana con fines comunicativos de primer orden; son generalmente orales y tienen que ver con

8 Pese a que se puede considerar como único cataclismo en la zona en el último siglo, los cataclismos se han reiterado con cierta frecuencia en el territorio nacional y la información mediática hace que las personas vivencien estos sucesos como propios, también se podría considerar la experiencia de desastres naturales de personas cercanas en la apropiación discursiva, es decir, relatar la tragedia del otro desde la experiencia personal y, así, re-memorizar y re-plantear la propia. la comprensión interna que se tiene de lo acontecido; son más cercanos a lo contextual por anclarse en un espacio y tiempo determinado'; se encuentran en diálogo con la experiencia y la territorialidad. Luego, al incorporar ciertas estrategias textuales por parte de los autores, se sitúan en el tránsito hacia un género discursivo secundario, se acercan a lo artístico y rearticulan en el discurso.

La realidad y la ficción suelen mezclarse y superponerse en el mismo discurso oficial frente a un evento, al punto que tal realidad se construye sobre la escenificación de mitos e invisibilización de ciertos sucesos, lo que implica ya un recurso de selección en el acontecimiento ${ }^{10}$. Sobre la carencia de una voz que esté fuera de esta oficialidad es que se instalan discursos comunitarios e intrahistóricos: discursos de la memoria y de las micromemorias que buscan rescatar los recuerdos y fijarlos (Mendoza, 2004) en una articulación significativa para la misma comunidad y sus integrantes.

El empleo que se haga del lenguaje en función de las estrategias narrativas es la fuerza que vehiculizará la visibilización de la textualización de la memoria. El lenguaje (la selección y el empleo que se haga de él) es una manera de re-descripción interpretativa (Presas, 1997) frente a la realidad cambiante e indefinible; de ese modo, funciona como instrumento de aprehensión de la realidad (o de la ficción, ya que hablamos de multiplicidad de discursos y multiplicidad de veces que se ha articulado tal discurso). El uso del lenguaje es, entonces, la herramienta que modela la capacidad narrativa del ser humano, le permite referirse a su entorno y sus acontecimientos, es función referencial:

Así, pues, si bien los textos de ficción "no reproducen los objetos existentes", sino que, "más bien proyectan sus objetos", ello no significará que realidad y ficción simplemente se opongan; sino más bien que la ficción es la estructura lingüística capaz de organizar la realidad de manera tal que

9 A diferencia de un género discursivo secundario que emplea teorizaciones, reflexiones de índole filosófico, existencialista, ontológicas, por mencionar algunos ejemplos.

10 Por ejemplo, las estrategias narrativas en la realización de los talleres. 
esta sea comunicable. Por ello, podemos decir ahora que, "en vez de ser su mero opuesto, la ficción nos comunica algo sobre la realidad" (Presas, 1997, p. 118).

En esta experiencia, la ficción narrativa empleada es la voz de la memoria tejida desde marcos sociales particulares a la comunidad estudiada, ella los articula a la luz de su ideario de mitificación y creencias; en definitiva, de la identidad que con el tiempo se ha ido conformando y de la necesidad de generar una autoidentidad en relación con el espacio que ocupan: el territorio.

\section{Códigos de la memoria social}

Barthes (2004) plantea dividir el texto para su análisis en varias unidades de sentido. Las unidades más pequeñas y que son base de la partición estructural se denominan lexías. Se trata de fragmentos contiguos que son unidades de lectura y para las que no se usa un criterio preestablecido en su división, se emplea el propio significante y el análisis del significado para constituir la mencionada unidad: "La lexía comprenderá unas veces unas pocas palabras y otras algunas frases, será cuestión de comodidad" (Barthes, 2004, p. 9).

La separación de las lexías se advierte determinada por las pausas lógicas del discurso y corresponde a las divisiones gramaticales: signos de puntuación, sintagmas, formas de coordinación o subordinación, etc. La misma separación gramatical implica un cambio en el sentido de lo enunciado, por tanto, la división del significado se va dando de forma implícita a la misma disociación en lexías.

Las lexías se separan en códigos que son "una especie de red, de tópico, a través de la cual se pasa el texto (o, mejor dicho: al pasar por él se hace texto)" (Barthes, 2004, p. 15). Estos códigos son cinco (las abreviaciones son tomadas del original): 1) hermenéutico (HER), 2) semántico (SEM), 3) simbólico (SIM), 4) proairético (ACC) y 5) Cultural (REF).

Los códigos que serán utilizados para el análisis de los relatos recogidos de la experiencia narrativa corresponden al semántico y al simbólico, por tratarse de los que ofician en la generación de la memoria como un marco social directo, es decir, están presentes en los géneros primarios de carácter oral constantemente. En tanto, los otros códigos pueden ser vistos como mecanismos de una textualización más sofisticada o secundaria, por lo que han sido dejados fuera del análisis. Pese a que la experiencia del taller entrega ciertas herramientas para aproximarse a un género secundario, más poético y de construcción más estilística, se trata de un primer acercamiento a este tipo de accionar textual para los sujetos escribientes.

El código semántico (SEM) adquiere valor desde las connotaciones que pueden tener las mismas palabras o sus relaciones dentro de la lexía, "llamemos a este elemento un significado (sin especificar más), o si se quiere un sema" (Barthes, 2004, p. 13). En el contexto interpretativo del corpus a analizar, este código es el que marcará la relación de lo acontecido y vivenciado con la tragedia sentida y textualizada, dado que el sema que guía las connotaciones de este código es la destrucción y el caos resultante. Posteriormente se define el código simbólico (SIM) como correspondiente a la diversidad en la construcción del texto y su relación con otros sistemas, es la pluralidad del texto, son significaciones dadas desde otras aristas socioculturales y que permiten entender la visión particular de un pueblo. Entonces, los elementos connotativos y plurales son los que articulan la memoria desde el entramado social que le da una moldura cultural.

Los códigos son vistos como voces constituyentes del texto y de la textualización, remiten a las voces anteriores (el texto mismo contiene la tradición cultural de la humanidad en las voces que lo configuran, el libro rizomático): "Se oyen voces en off: son los códigos [...]: la concurrencia de las voces (los códigos) deviene escritura, espacio estereográfico, donde se cruzan" (Barthes, 2004, p. 16).

\section{Aspectos generales de la intervención}

\section{Metodología}

En coherencia con el propósito del estudio, se trabajó una metodología cualitativa de alcance 
descriptivo que permitiera analizar el ejercicio de la memoria desde la mirada particular que poseen sus protagonistas. De este modo, se utilizó la narración y conversatorio para propiciar la emergencia de la memoria de la catástrofe y un taller de narrativa en el que se impulsó la práctica escritural sobre el evento, en el cual se produjeron los relatos testimoniales. Además, se utilizó como técnica la entrevista en profundidad como un medio adecuado para acceder a la experiencia de construcción de la memoria desde el punto de vista del grupo de sobrevivientes participantes del taller. Las entrevistas fueron individuales con preguntas abiertas; se realizaron $10 \mathrm{y}$ se focalizaron con participantes de la intervención. Durante las sesiones de entrevista se desarrolló una conversación libre con cada participante bajo el tópico del ejercicio de la memoria, para así no solo abordar el resultado de la memoria como constructo textual, sino incorporar la visión y opinión de los participantes y escribientes en el proceso experimentado durante el desarrollo del taller.

\section{Descripción de los participantes}

Previo al desarrollo del taller, un grupo conformado por psicólogos y sociólogos de la comuna y del Programa Servicio País determinó el grupo que participaría del taller según los siguientes criterios: a) haber vivenciado in situ el terremoto de 1960, b) pertenecer a sectores rurales de la ribera del río Maullín y c) manifestar interés en realizar el taller y conversatorio.

Los sujetos participantes en este estudio son 15 adultos mayores, 9 mujeres y 6 varones; del total, 4 tienen enseñanza básica completa, 7 enseñanza media y 4 formación técnica, en tanto que sus edades van desde los 59 hasta los 70 años de edad ${ }^{11}$. Dado el grado de afección del terremoto que abarcó a la población de Maullín, y en especial el sector La Pasada por su cercanía a la ribera del río Maullín,

11 De acuerdo con el año de nacimiento registrado en las cédulas de identidad de los participantes, 2 personas contaban con menos de 60 años, ya que fueron inscritos en el Registro Civil después de los 6 años de edad, como era usual en el pasado y especialmente en los sectores rurales. Teniendo estos antecedentes en cuenta se determinó que estos vecinos sí podrían participar del taller en cuanto podían rememorar el evento del terremoto y tsunami. la muestra se orientó a sobrevivientes que provinieran de estos sectores, para que pudieran entregar una mirada completa sobre el habitar el mismo territorio que fue azotado por la tragedia.

Los participantes provienen entonces del mismo lugar y al momento de ocurrido el acontecimiento algunos eran niños y otros adolescentes, o adultos jóvenes con familias conformadas. Estos escribientes produjeron, durante el taller de narrativa y conversatorio, los relatos analizados en este estudio.

\section{El taller de narrativa y conversatorio}

La intervención consistió en un taller de narrativa que duró 10 sesiones desarrolladas entre agosto y diciembre de 2015 con una duración promedio de una hora y media. Las sesiones se desarrollaron sistemáticamente en la Biblioteca Dorila Águila Aguilar de Maullín, durante los días viernes o sábado, según la disposición horaria de los participantes.

Estas sesiones se realizaron en el marco del proyecto "Relatos del Territorio" iniciativa financiada por la Fundación para la Superación de la Pobreza y desarrollada en conjunto con académicos y estudiantes de la Facultad de Educación de la Universidad San Sebastián. El proyecto buscaba abordar la problemática de la falta de entendimiento comunitario e invisibilización de conocimientos locales y memorias sociales en la gestión de riesgo de territorios vulnerables que han estado expuestos a desastres naturales.

Todas las sesiones se realizaron con un intervalo de dos semanas; los asistentes se fueron familiarizando con las estructuras narrativas, realizaron ejercicios escriturales de producción; analizaron, explicaron, editaron y corrigieron con la contribución de todos los sujetos y de los investigadores a cargo.

La primera sesión tuvo como objetivo que los integrantes del grupo pudieran familiarizarse en la conformación del grupo taller y como actividad de emergencia de la memoria se empleó la conversación y lectura de textos periodísticos que abordaron la tragedia en esa época para contrastar con la memoria de los participantes e ir trazando lineamientos de los intereses comunicativos de cada 
escribiente. Luego, entre la segunda y sexta sesión se trabajaron estrategias de construcción del relato testimonial a través de lectura de modelos, generando esquemas de diseño de la estructura narrativa y generación y corrección de borradores, para transitar paulatinamente, entre la sesión séptima y novena a la producción del relato final, mediante la recopilación de información referencial sobre el terremoto y tsunami surgidos en la puesta en común durante los conversatorios. La sesión diez implicó la presentación de los relatos en una ceremonia de cierre del proyecto en la que participaron invitados de la comunidad y familiares.

El acceso a la memoria y su devenir fue motivado mediante un conversatorio que se realizó entre cada taller, en esta instancia de charla y cercanía en la biblioteca, las personas nos fueron contando cómo se desenvolvían en su contexto ambiental y sociocultural antes y después del terremoto, ejercicio que ayudó a ir mapeando la memoria del evento. Esta estrategia fue la más adecuada para adentrarse en los terrenos de la memoria, que muchas veces se encuentra en el interior de cada uno como un cúmulo de imágenes desordenadas producto del trauma que implica la catástrofe. Al conversar y compartir, los participantes fueron articulando un discurso y testimonio desenvuelto, evitamos de este modo el choque y el encasillamiento que puede suponer la interrogación, método al que no están acostumbrados. Mediante la modalidad de conversatorio propiciamos un contexto que recreara situaciones cotidianas con la complicidad de un mate, en las que la conversación se transforma en un pasatiempo. De estos conversatorios se fue tejiendo la memoria del evento, sin estructura, sin guion predeterminado; emergió como recuerdo libre.

\section{Procedimiento de análisis}

El análisis de los relatos se realizó siguiendo el modelo de análisis textual propuesto por Barthes en $s / z$ (2004). Los relatos fueron separados en lexías y luego estas agrupadas en los códigos del modelo, como fue aclarado anteriormente, se tomaron solo dos códigos por su pertinencia para la constitución de los marcos sociales señalados: el código semántico y el código simbólico. Dentro de estos códigos, se identificaron subcódigos con los que se realizó la interpretación.

Esto obedece a una metodología complementaria que mezcla aspectos deductivos e inductivos, es decir, se parte de categorías predeterminadas que se evidencian en los textos analizados (la organización de las lexías en códigos) y desde cuyo análisis surgieron otras categorías (subcódigos) no contempladas previamente que se explicarán y ejemplificarán más adelante en el apartado de resultados. Los subcódigos nacieron al alero de la categoría de filiación y para su separación se empleó un criterio de sentido asignado por las palabras y sintagmas constituyentes de la lexía en cuestión.

En el caso de la información recogida mediante entrevistas la reducción de datos se realizó a través de segmentación de unidades y categorización. El criterio que se utilizó para dividir la información en unidades fue el criterio temático según la información que aportó cada participante en particular. Se empleó un sistema de categorías emergentes mediante procedimiento inductivo con el cual se analizó cada categoría relacionándola con subcategorías y con otras categorías (Taylor y Bogdan, 1990).

\section{Resultados}

En primer lugar, se presentan los resultados sobre los significados culturales y simbólicos que operan como marcos sociales en los relatos testimoniales. En segundo lugar, se presentan los resultados organizados por categorías y subcategorías provenientes del análisis de contenido de las entrevistas realizadas a los participantes del taller con el objetivo de describir la experiencia de construcción, deconstrucción y reconstrucción de la memoria mediante la narración.

\section{Marcos sociales de la memoria}

El corpus de análisis está constituido por el conjunto de relatos producidos en el marco de la realización de los talleres de narrativa. Cabe señalar que a pesar de que se analizaron todos esos textos, en los resultados se muestran solamente lexías 
representativas de los códigos y subcódigos y que resultaban más pertinentes para su análisis.

La extensión de los relatos en cuestión varía entre una y tres páginas, en la mayoría se emplean recursos narrativos asociados al taller aunque de orden primario. El posicionamiento del narrador va desde la actualidad con una mirada hacia el pasado y desde el pasado mismo transitando hacia el presente en el final, este movimiento genera relatos enmarcados en el límite de la intrahistoria.

Como una forma de facilitar la referencia a los textos analizados, utilizaremos la siguiente simbología para aludir a los datos examinados: $\mathrm{T}$ (número) para el texto y (número) para la lexía con la que se ejemplifica el subcódigo.

\section{Código semántico}

Este código que ha sido señalado como uno de los marcos sociales constitutivos de la memoria comprende cuatro subcódigos, estos son: caos, maldición/catástrofe, arraigo/familia y nuevo orden. Se ofrecerán, como ejemplo, algunas lexías representativas y luego se comentará el sentido de cada subcódigo.

\section{Subcódigo "Caos"}

(a) T4 1 Nada se podía entender más que caos; (b) T4 6 Lo que antes era mi casa, mi morada, ahora no era más que un montón de escombros sin sentido;

(c) T5 4 Sentí que mi vida y esperanzas se los llevó esa agua avasalladora.

Este subcódigo nace del desorden que se genera en una situación de orden previo a la catástrofe, se relaciona con la perplejidad de los habitantes de un territorio que han visto quebrada la disposición que les permite entenderse desde los elementos simbólicos que los constituyen en tanto comunidad.

\section{Subcódigo "Maldición/catástrofe"}

(a) T7 2 Esto parecía una maldición "¿Por qué?" me preguntaba entre sollozos; (b) T7 3 Una nube de polvo y dolor cubría todo lo que antes había sido mi pueblo; (c) T7 4 La noche caía como el futuro, negro y desolador.
El quiebre generado por el evento cataclísmico hace percibirse a los habitantes del territorio como sobrevivientes malditos en medio de elementos que dan cuenta de tal maldición patentizada por la catástrofe.

\section{Subcódigo "Arraigo/familia"}

(a) T5 3 Tenía que seguir adelante por sus hijos, y más por ese hijo que crecía en su interior; (b) T5 6 Hoy mis hijos se sienten orgullosos de que pudimos superar esos duros momentos; (c) T5 8 Hasta en esos negros momentos del año 60 no perdí de vista que sería abuela algún día y a ellos le contaría cómo reconstrui mi hogar y mi vida.

Uno de los elementos que permite dar cuenta de la existencia de la comunidad y que, al mismo tiempo, los hace seguir aferrados a la territorialidad es la familia. Sin esta, no podría entenderse el ímpetu de los sujetos de continuar adelante y de superar la condición de malditos por la catástrofe.

\section{Subcódigo "Nuevo orden"}

(a) T4 2 Como pudimos comenzamos a limpiar lo que quedó de nuestras pertenencias; (b) T4 $3 \mathrm{~A}$ veces le cuento a sus nietos la desesperación que sentí en ese momento; (c) T4 7 Un nuevo pueblo se levanta hoy sobre las ruinas que dejó el mar.

Orden, des-orden y finalmente nuevo orden para re-habitar y re-configurar un territorio y una comunidad. Los sobrevivientes son capaces de rearticular su devenir y de levantarse ante la catástrofe; no obstante, lo ocurrido deja una marca emocional profunda en sus vidas y en las de sus descendientes.

\section{Código simbólico}

El segundo de los códigos barthesianos que se ha tomado para este estudio incluye nueve subcódigos que han sido rastreados en los relatos del corpus, a saber: memoria, una fuerza superior, la tierra: una tumba, la protección, sobrevivientes, el agua que destruye, vida y muerte confundidas, el desarraigo, el lamento. 


\section{Subcódigo "Memoria"}

(a) T6 1 Miro a mi alrededor, todo lo que se ha logrado construir es una forma de recordar el terremoto y también de ver nuestro porvenir; (b) T8 3 Recordar esa situación me hizo llorar un poco antes de hablar; (c) T2 26 Las personas que hoy habitan este pueblo, los descendientes no saben la tragedia que significó ese día.

La memoria es una entidad guardada en el interior de las personas, se activa con una situación específica y se devela como un recuerdo lleno de carga emocional y afectiva en relación con los sucesos vividos. Al mismo tiempo, la memoria es portadora de una idea de porvenir, de cómo a partir de un pasado se puede articular un futuro, la memoria es la narración del pasado que busca continuar plasmándose en sucesos de narraciones venideras.

\section{Subcódigo "Una fuerza superior"}

(a) T4 9 Pedía y pedía a Dios que acabara el terremoto; (b) T5 5 Todos pedíamos a Dios que se apiadara de nosotros, que por favor pasara ya; (c) T7 7 Ella decía entre llanto: “Por qué merecemos esto Señor?”.

La catástrofe es asumida como un castigo emanado de dios, al mismo tiempo, el amparo para sobreponerse a tal momento también descansa en la deidad y en los rituales que se pueden ofrecer como mecanismo dialógico con dios.

\section{Subcódigo "La tierra: una tumba"}

(a) T4 10 Maullín había sido tragado por la tierra al parecer; (b) T7 13 De los desaparecidos solo se sabía que se los había tragado la tierra; (c) T8 13 Ahora todo el pueblo parecía un cementerio.

La concepción de la muerte se relaciona con la tierra como elemento que da la muerte y a la vez es contendor de esta. Si la tierra es la tumba en la que todo ser humano terminará, en este momento de catástrofe del evento señalado ella oficia de una potencial tumba de toda la comunidad.

\section{Subcódigo "La protección"}

(a) T8 4 Nos agarramos las manos entre todos mientras pasaba el temblor; (b) T7 5 Los niños lloraban aferrados a la falda de su madre; (c) T8 5 Apenas pareció terminar el movimiento los padres corrieron a buscar a sus hijos.

Ante la catástrofe y la incertidumbre de lo venidero, la protección se hace necesaria como un elemento para poder continuar con la supervivencia de la comunidad; la protección se da en sentido microcomunitario, es decir, dentro de la familia los mayores y más fuertes ofrecen la protección a los menores y más débiles.

\section{Subcódigo "Sobrevivientes"}

(a) T6 5 Todos se agarraban de los árboles que estaban cerca; (b) T6 8 No me acordé que estaba sin zapatos, solo corrí hasta lo más alto del cerro sin pensar en las espinas y en las zarzamoras; (c) T7 12 Fue tanto el miedo al agua que subía por el pueblo que sin saber cómo llegué hasta el techo de la casa.

La sobrevivencia en un momento de catástrofe significa un actuar desde los instintos, no se puede planear el accionar ni mediar pros y contras en relación a lo que se debe hacer.

\section{Subcódigo "El agua que destruye"}

(a) T4 14 El río irrumpió sin piedad entre las casas, se llevó todo, si hasta cadáveres dicen algunos que vieron; (b) T3 16 El agua abrió las tumbas y sacó los restos de los muertos de su descanso eterno; (c) T1 10 El río que siempre nos dio de comer y de vivir hoy día nos quitaba todo y amenazaba con la muerte.

Un elemento de destrucción importante, incluso más que la tierra, es el agua. Con ello se da cuenta de la fuerza que tuvo el tsunami al momento de arrasar con la comunidad aludida. La fuerza destructora del agua no respeta, pasa por sobre lo que constituye la territorialidad concreta (casas, construcciones, espacios) y simbólica (hogar, lugares sagrados como iglesias o cementerios) que son espacios de construcción de la memoria. 


\section{Subcódigo "Vida y muerte confundidas"}

(a) T3 17 Los muertos anteriores flotaban junto con los muertos de ahora; (b) T8 19 La comunidad tuvo que separar los muertos anteriores con los muertos recientes para que el cura les pudiera dar su última bendición; (c) T1 17 Se cree que hubo más ahogados ese día que todos los muertos del cementerio.

Este subcódigo representa un momento escatológico para la comunidad, confundir la vida con la muerte quiere significar el fin, un fin que viene de los elementos de la naturaleza, como se presenta en los relatos proféticos de muchas culturas.

\section{Subcódigo "El desarraigo"}

(a) T4 11 La fuerza del agua separó a muchos que no alcanzaron a hacer nada; (b) T7 9 El agua arrasó con todo, hasta con mis esperanzas; (c) T2 12 Mis ojos solo veían destrucción y soledad, pero no lloraba.

La devastación del territorio que se habita representa y es un desastre para la comunidad, la desolación del paisaje no permite ni siquiera pensar en un futuro, funciona como un elemento psicológico de bloqueo emocional que puede devenir en un bloqueo en el actuar.

\section{Subcódigo "El lamento"}

(a) T4 12 Sus lamentos hacían que sus pequeños hijos lloraran más y más; (b) T2 19 La amargura marcó su rostro por mucho tiempo; (c) T6 3 Lloraba porque no sabía cómo alimentaría a su familia en los días venideros.

La comunidad afectada se siente desprotegida y a la deriva, lo que se traduce en un lamento cargado de dolor, tristeza, desconsuelo, no poder entender y no vislumbrar salida.

\section{La experiencia de reconstrucción de la memoria}

Se proporciona, en primer lugar, una breve descripción de cada categoría para luego mostrar ejemplos de su manifestación. El sistema utilizado para identificar los fragmentos de las entrevistas es: $\mathrm{R}$ para referirnos al número de registro de la entrevista y $S$ para nombrar al sujeto participante del taller.

\section{Categoría "Memoria como ejercicio social"}

Esta categoría refiere a la textualización del evento como un constructo social y cultural que la comunidad discursiva erige a partir de patrones simbólicos y culturales con los que se busca dar verosimilitud al relato y construir una memoria colectiva. Dentro de esta categoría general se incluyen tres subcategorías: ficcionalización del relato, rearticulación de micromemorias, identidad $y$ territorio.

\section{Subcategoría "Aspectos ficcionales del testimonio"}

Mediante las entrevistas fue posible conocer de qué forma se articulan y entremezclan la realidad y la ficción en el discurso comunitario y cómo se construye en la interacción del grupo el correlato del discurso oficial o más bien dicho, el discurso intrahistórico.

\section{(a)}

R2 P6: $O$ sea [...] si uno mira con lo que empezamos y con los que terminó el relato que escribí [...] hay diferencias. Porque me fui acordando de otras cosas que pasaron y después también con lo que nos enseñaron los profes lo fui aplicando para mejorar lo que escribía, pero siempre manteniendo lo que recuerdo, porque si no, no sería verdad [...]

R4 P2: Por ejemplo, cuando uno le da el inicio para que sea más interesante o en las características de los lugares o de las personas que uno va relatando [...] que sea algo más rápido de leer, porque si no va a ser más enredado o nadie lo va a tomar en cuenta.

Subcategoría "Rearticulación de las micromemorias"

(b)

R5 P3: Uno sabe lo que pasó [...] lo que uno se acuerda [...] pero también en los talleres me fui acordando de otras cosas que pasaron y que me había olvidao, porque algunos éramos más chicos $y$ no sabíamos todo lo que pasaba y otros vecinos 
de acá del taller tenían más edad en ese momento y se acuerdan de otras cosas.

R2 P8: Algunas partes del testimonio era de lo que yo me acordaba y otras me fui acordando según lo que dijeron los vecinos, porque como pasó tanto tiempo [...] habian cosas olvidaas y ahí una dice: claro que sípues.

\section{Subcategoría "Identidad y territorio"}

\section{(c)}

R2 P3: Quería aprender cosas nuevas [...] por eso me metí en el grupo. Porque hace rato que ya dejé la escuela, pero siempre me gustó leer y también poder juntarnos con los vecinos [...] lo pasamos bien. Es importante poder dejar este testimonio en papel pa' que nuestros nietos no se olviden [...] Fíjese que mis papás no quisieron dejar el campo nunca, somos de acá, acá vamos a morir, en la misma tierra que se partió.

Tal como se aprecia en el registro (a), el traspaso de las técnicas narrativas posibilitó el paso de un género discursivo primario a uno secundario, en el que se articula realidad y ficción a partir de la conformación de una macromemoria como se expresa en el registro (b). Con ello se conformó el discurso comunitario en la articulación de memorias y micromemorias encuadradas en los marcos sociales seleccionados por la comunidad. Es decir, el taller y el ejercicio de la memoria permitió comprender los acontecimientos, completarlos y enriquecerlos a la vez que la incorporación de estrategias textuales permitió redescribir la realidad y la ficción. Esta articulación se encuadra en códigos culturales y simbólicos relacionados con el ideario de mitificación y creencias de la comunidad y su identidad vinculada al territorio del que son parte como se indica en el registro (c). En efecto, cuando las personas evocan el evento, construyen su identidad y levantan su propia historia.

\section{Categoría "Memoria como ejercicio afectivo"}

La memoria es resignificada no solo a partir de lo que se recuerda, sino también sobre cómo se recuerda. Esta categoría aborda el modo en que se fija la experiencia a partir de los significados, emociones y de la comprensión interna que se tiene de lo acontecido. Dentro de esta categoría se incluyen las siguientes subcategorías: emergencia de la memoria, elementos sacros y escatológicos, desterritorialización.

\section{Subcategoría "Emergencia de la memoria"}

La memoria emerge principalmente a través de la pregunta “¿qué ocurrió?". Remonta a los sujetos a un pasado que se hace presente y se va deconstruyendo y reconstruyendo en un espacio y tiempo cargado de significados. Como lo expresa el siguiente participante al preguntarle cuándo surgió el recuerdo:

(a)

R9 P5: Cuando supe de este taller en la biblioteca, me acordé altiro [...] algo rápido eso sí [...] Después cuando empezamos el taller, el primer día estuvimos conversando y uno de los profes me preguntó: “¿Cómo lo vivió ese momento?”. Algo así me preguntó [...] Ahí volvió la memoria, cuando era chico y pasábamo' el río pa' ir a la escuela en el pueblo [...] ese día estaba tan bonito y después se vino la noche no ma' [...] escuchaamos la pura llantería no ma'.

Sin embargo, también emerge la memoria a través de las imágenes como lo señala P3:

\section{(b)}

R7 P3: $;$ Ooy! [...] Cuando vimos las fotos y videos sobre el terremoto, fui acordándome de lugares $y$ sobre todo como quedó destruido [...] sabe que uno se acuerda cómo quedó todo, pero al ver una foto, por ejemplo, me doy cuenta de que fue más grande de lo que uno alcanza a acordarse incluso.

\section{Subcategoría elementos sacros y escatológicos}

La memoria es un ejercicio cargado de interpretaciones y de simbolismo. Mediante las entrevistas es posible apreciar que la memoria selecciona y recrea los acontecimientos significativos en función de la filiación a la tradición religiosa, valores y afectos como se expresa a en (a) y (b): 
(a)

R3 P1: Nosotros que sufrimos este cataclismo pensamos que era como una maldición de Dios ¿sabe?, un castigo divino y por eso clamábamos a Dios también para que nos salvara de la tragedia, pudiéramos encontrar a nuestros familiares [...]

(b)

R1 P10: [...] uno recuerda cómo vivió el asunto $y$ por eso puede ser que todos tengamos recuerdos distintos [...] aunque venimos del mismo sector, acá somos too vecinos [...] Bueno [...] algunos estaban acá en el pueblo cuando pasó y otros en el campo, pero igual la experiencia es distinta independiente del lugar, depende de la pena [...] rabia o miedo con el que uno se acuerde [...]

Al mismo tiempo se destacan elementos escatológicos en la urdimbre de la memoria como lo expresa P5:

(c)

R1 P5: De lo que más me fui acordando en el taller, junto con lo que ibamos conversando con los vecinos [...] era el tema de lo oscuro [...] y el agua! [...] que venía negra como en el fin del mundo, como que venía la muerte en forma de agua [...] eso nos pasó a todos yo creo. Una ola y otra ola, el mar junto con el río, algunas casas bajaban y otras subían, por la corriente pue'.

\section{Subcategoría "Desterritorialización"}

El reconocimiento que los sobrevivientes hacen sobre el despojo del territorio durante el cataclismo es un elemento afectivo que los aproxima a la posibilidad de acercarse al recuerdo y a la memoria como lo señala el siguiente participante al preguntarle el momento del catastro posterior a la bajada del agua:

(a)

R3 P8: El momento más fuerte [...] al menos para mí, fue cuando vimos lo que había quedado [...] porque por el agua y estaba oscuro [...] cuando bajó el agua la tierra estaba como hundida, no teníamos nada, solamente lo puesto, algunos sin familia, imagínese.
Según lo expresado por los participantes, el taller posibilitó la construcción del recuerdo para dejar un legado a las generaciones actuales y futuras sobre la hazaña de aquellos que decidieron quedarse y reterritorializar el espacio despojado.

(b)

R4 P6: $; U u y !$, muchas cosas [...] pero lo que más me queda de esta experiencia son los relatos que podemos dejar como testimonio, no tanto de la tragedia [...] porque hay muchas tragedias hoy en día [...] yo creo que con los relatos estos niños, jóvenes se van a dar cuenta que este pueblo existe porque no nos fuimos a otro lao [...] volvimo' a levantar las casas y aquí estamos.

(c)

R3 P4: [...] dejar en la memoria las cosas que están como olvidá' ya [...] porque los jóvenes ven este desastre como una historia pasada y no le toman el peso. Yo creo que cuando lean nuestros cuentos van a darse cuenta y valorar lo que tuvimos que pasar [...] formar familia [...] y ahora ellos están acá también.

\section{Categoría "Memoria como ejercicio político"}

El proceso de reconstrucción de la memoria es también un ejercicio político puesto que la comunidad selecciona y recrea lo que es significativo en términos de las concepciones morales, religiosas e identitarias que quiere destacar en su proyecto para habitar un territorio específico. La subcategoría que se incluye es "Proyecto comunitario: el legado".

\section{Subcategoría "Proyecto comunitario: el legado"}

Estos actores sociales seleccionan los eventos y la forma de narración con que serán comunicados a las futuras generaciones y que, además, determinarán la forma en que serán influenciados relatos presentes, venideros u otros del pasado que se quieran visibilizar, como una manera de anclar el proyecto de la comunidad en los marcos sociales.

(a)

R1 P7: Cuando fuimos cada uno diciendo lo que vivió, contando su experiencia [...] la forma en 
que cada uno le da importancia o cómo lo vivió puede ser distinta, pero hay cosas que nos unen a todos [...] es como que fuera un solo testimonio de todos acá, uno iba diciendo una cosa y el otro otra y así uno se iba acordando también, de tanto gente que murió.

R2 P5: Lo que se ve en todos los relatos de los vecinos es la sobrevivencia [...] eso creo yo [...] la valentía de nuestra generación, porque todos formamos nuestras familias, nos casamos, tuvimos hijos y nietos que van a heredar esto. Aparte, ¿qué les podemos dejar? Que quede una enseñanza al menos [...] tienen que cuidar este lugar, el mar se enoja [...] estar en comunión con Dios que es el único que castiga y perdona.

Por último, los códigos y subcódigos permiten entender la configuración de los textos desde los patrones social-moral-cultural que sustentan a la comunidad estudiada, por lo que se puede decir que las líneas de fuga que operan en la construcción, deconstrucción y recontrucción del proceso de la memoria van desde la evocación, el recuerdo, hasta la generación de un discurso conformado en conjunto (taller y comunidad).

\section{Conclusiones}

Este estudio ha tenido como objetivo aproximarnos al conocimiento de la ideación del pasado mediante el ejercicio de la memoria y analizar qué significados culturales articulan la resignificación de las representaciones simbólicas de las vivencias del terremoto y tsunami de 1960 desde la experiencia de un grupo de sobrevivientes a esta catástrofe. Se ha utilizado una metodología cualitativa a través de un taller de narrativa, conversatorio y entrevistas en profundidad a partir de la cual se analizaron los relatos testimoniales producidos por los participantes del taller y además sus discursos en torno al proceso de construcción de la memoria.

Los resultados entregan datos que se orientan desde dos perspectivas complementarias y que permiten entender esta vivencia particular de los escribientes/sobrevivientes, a partir de la reflexión colectiva y personal en un ámbito discursivo conciliador, de entendimiento y, a la vez, de coconstrucción del recuerdo/memoria.

Para aclarar estos puntos iniciales, se debe referir que las dos ópticas mencionadas, que son las tomadas en los resultados y análisis de los datos, tienen que ver con un producto y con una mirada al proceso. Por un lado, se cuenta con el corpus de textos narrativos generados en el taller (es decir el producto), y por otro, se tienen las respuestas de las entrevistas que ofician como motor reflexivo (esto es: la mirada al proceso), tanto de la experiencia del taller, de las técnicas narrativas aprendidas, como del desarrollo colectivo de construcción de una memoria. En este sentido, se trata de un trabajo colaborativo y comunitario, que se ampara bajo la existencia de un marco social de la memoria común como punto de partida del taller: re-mirar, re-visar, re-entender y re-contar y re-escribir lo acontecido/ vivenciado durante el cataclismo del año 60. Esta visión en "re" (revisión) es articulada textualmente a través de los patrones simbólicos y culturales específicos de la comunidad aludida, para generar una discursividad específica y característica (de la misma comunidad) en torno al recuerdo testimonializado, es decir, la memoria en formato texto.

El taller opera como filtro que genera el paso de un género de primer orden a un género de segundo orden, de la cercanía del relato oral se ha pasado al relato escrito bajo parámetros genéricos específicos, en el caso de los relatos testimoniales, mientras que en las entrevistas se puede hablar de un relatodescriptivo de la construcción activa de la memoria y de la intersubjetividad en la que se configura, razón por la que las categorías que emanan de ellas se organizan desde "la experiencia de construcción, deconstrucción y reconstrucción de la memoria del evento", que en definitiva se relacionan con el cómo del recuerdo, ya sea seleccionar, organizar o replantear los recuerdos, así como la creación y cambio de significados en torno a ellos.

La forma en que se entrega el mundo reconstruido-narrado sigue una gramática del caos que pretende dar cuenta de un contexto de destrucción y desastre, esta misma forma de textualizar el evento es 
como ha pasado de voz en voz (multiplicidad de voces), desde los antepasados hasta las generaciones actuales, o también como los supervivientes lo han recogido de otros supervivientes, por lo que se puede hablar de una herencia léxica en torno al cataclismo: la memoria del evento textualizada con una gramática del caos.

Tal herencia léxica ha sido portada por un yomúltiple que aúna la multiplicidad de voces de las que se habla en los relatos y el taller, al socializar su experiencia, de manera intencional o no, los emisoresescribientes llegan a punto común que es expresado por la voz de los narradores de los relatos y la de los escribientes. Esta postura se comprueba no solo mediante los relatos recogidos, sino también apoyado en lo expresado en las entrevistas.

Cabe destacar que los relatos que configuran el corpus son la metatextualización, son el texto formal escrito (producto del taller, con las implicancias técnicas que ello tiene) del relato oral-original que es la primera memoria, esto implica que el ejercicio escritural es rememorar la memoria del evento, el traer a la luz los ecos de la memoria. Mientras que las entrevistas ofician de la misma manera, pero en un sentido fuera de lo escritural y más centrado en la reflexión acerca de la experiencia.

En relación con los códigos simbólicos y culturales que operan como marcos sociales de la memoria, se aprecia en un comienzo el castigo, la salvación y la clemencia bajo el alero de la configuración cristianocatólica, por lo que se puede entender la presencia de elementos sagrados para la comunidad, elementos de los que depende la salvación o la condena en este caso específico (subir el cerro implica salvarse, el agua destruye, arriba es el espacio sagrado mientras que el agua adquiere connotaciones bíblicas), lo que nuevamente es retomado en las entrevistas, ya que en una de ellas se menciona: "Es Dios quien castiga y perdona”. Al respecto, esta visión simbólica y cultural también se plasma en la gramática del caos, dado que se deja manifiesto el temor y clamor a dios.

Por otro lado, se observa la presencia de la familia, la sobrevivencia y el apego al lugar como la simbolización del legado a las generaciones presentes y futuras del territorio. Cabe señalar que esta manera de simbolizar tiene como objetivo la organización territorial en torno a unidades funcionales, de parentesco y afectivas, se relaciona directamente con la sobrevivencia ante eventos catastróficos y de zonas aisladas (los muertos perdidos pueden estar en esa tierra o en ese mar, rehabilitar y rehabitar ese territorio lo transforma de maldito en sagrado: el hogar). Los sobrevivientes generan un legado que sienten como frágil ante el olvido y la migración a centros urbanos más grandes (los descendientes desconocen cómo se vivió y superó la tragedia). El territorio es la base de subsistencia de las personas y por lo mismo la base de la organización y forma de vida familiar, la ideación de un pasado, presente, futuro y forma de vida tiene directo alcance con el cómo se habita y cómo se vivencia, por ello el valor de visionar, tanto hacia el pasado como hacia el futuro a través de un legado.

Desde el análisis de las entrevistas realizadas a los sujetos participantes del estudio, se desprende que el proceso de reconstrucción de la memoria es un ejercicio social en tanto lo que se recuerda es una construcción intersubjetiva que recoge un pasado producido y mantenido socialmente.

El ejercicio de traer sucesos a la memoria implica una construcción, deconstrucción y reconstrucción del evento, el material guardado en la memoria debe verbalizarse con nuevas herramientas que se han incorporado en el devenir de la vida (las técnicas narrativas son un caso bastante específico y de fácil rastreo en esta experiencia). Primero, se puede aludir a la propia experiencia, luego a la experiencia en voces de otros supervivientes, a la rearticulación de recuerdos, a querer construir el recuerdo de una manera más poética. Esto quiere decir que se puede entender una multiplicidad de voces, incluso considerando al mismo sujeto escribiente involucrado, pues no es el mismo al vivenciar el evento, al recordarlo y al textualizarlo, es un sujeto devenido, un sujeto en constante modificación.

Por otra parte, es también un ejercicio afectivo. Recordar es una actitud cargada, antes del recuerdo llega un afecto, una emoción que evoca el recuerdo 
y que hace emerger la memoria. Los afectos y sentimientos incitan la búsqueda de sentido, es el compromiso afectivo lo que hace de los momentos algo memorable. Además, la reconstrucción subjetiva e intersubjetiva del recuerdo se vale de herramientas simbólicas y culturales que permiten comprender y otorgar sentido al pasado, interpretando y trayéndolo al presente, en este caso la filiación a la tradición religiosa, valores y afectos de la comunidad con el tamiz por el que se da sentido a las rememoraciones individuales.

Como ejercicio político, el continuo quiebre del texto es el acto que hacen los sujetos escribientes una y otra vez para generar una discursividad en torno al evento y así moldear la memoria múltiples veces y de múltiples formas. Por tanto, se entiende que la memoria es una entidad viva, activa y deviniente dentro de la comunidad, además la perfila y direcciona. La comunidad es, en definitiva, lo que se ordena desde la triangulación memoria, territorio y cultura (estos dos últimos conceptos correspondientes a los marcos sociales que generan la forma de la memoria).

Con todo, los sobrevivientes ven la necesidad de estructurar la memoria del evento como una entidad viva en el habitar ese territorio, ya que muchos descendientes pueden olvidar y así, no considerar la particularidad del lugar en donde viven.

De este modo, los sobrevivientes simbolizan el territorio que habitan como un objeto de representación en el cual también han desarrollado un apego afectivo. En este caso se puede hablar de pertenencia socioterritorial a partir de la experiencia traumática en este territorio en la cual los sobrevivientes han incorporado simbolismos propios del lugar.

\section{Referencias}

Atwater, B., Cisternas, M., Yulianto, E., Prendergast, A., Jankaew, K., Eipert, A., Starin, F., Warnakulasuriya, I., Tejakusuma, I., Schiappacasse, I. y Sawai, Y. (2013). The 1960 tsunami on beach-ridge plains near Maullín, Chile: Landward descent, renewed breaches, aggraded fans, multiple predecessors. Andean Geology, 40(3), 393-418. Recuperado de http://www.scielo.cl/scielo.php?script $=$ sci arttext\&pid=S0718-71062013000300001\&lng=es\& tlng=en

Bajtín, M. (1999). Estética de la creación verbal. México: Siglo Xxi Editores.

Barthes, R. (2004). S/Z. Buenos Aires: Siglo xxi Editores.

Beverley, J. y Achugar, H. (1992). La voz del otro, testimonio subalternidad y verdad narrativa. LimaPittsburgh: Latinoamericana Editores.

Bruner, J. (1990). Actos de significado. Más allá de la revolución cognitiva. Madrid: Editorial Alianza.

Candau, J. (2008). Memoria e identidad. Buenos Aires: Ediciones del Sol.

Chávez, J. (2007). Qué lejos estoy del suelo... apuntes sobre el espacio, el territorio y la memoria. Territorio, Patrimonio y Ciudad. Cuadernos de Trabajo, 4, 30-38. Recuperado de http://www.sociologia.uson.mx/docs/ publicaciones/cuadernodetrabajo/cuaderno4.pdf

Corti, P., Moreno, R. y Widow, J.L. (2011). Historia, memoria y narración. Viña del Mar: Ediciones Altazor.

Halbwachs, M. (2004). La memoria colectiva. Zaragoza: Prensas Universitarias de Zaragoza.

Jelin, E. (2002). Los trabajos de la memoria. Madrid: Editorial Siglo XxI.

Maskrey, A. (1993). Los desastres no son naturales. Bogotá: La Red-Tercer Mundo Editores.

Martínez, M., y Scheffel, M. (2011). Introducción a la narratología: Hacia un modelo analítico descriptivo de la narración ficcional. Buenos Aires: Las Cuarenta.

Mendoza, J. (2004). Las formas del recuerdo. La memoria narrativa. Athenea Digital, Revista de Pensamiento e Investigación Social, 6, 1-17. Recuperado de http://www.raco.cat/index.php/Athenea/article/ view/34157/33996

Nora, P. (2001). Entre mémoire et histoire. En P. Nora (dir.), Les lieux de mémoire (pp. 23-43). París: Gallimard.

Lynch, G. y Oddone, M.J. (2008). Las memorias de los hechos sociohistóricos en el curso de la vida. Revista Argentina de Sociología, 6(10), 121-142. Recuperado de http://www.redalyc.org/articulo.oa?id=26961009

Pinilla, A. (2011). La memoria y la construcción de lo subjetivo. Revista Folios, 34, 15-24. Recuperado de http://www.redalyc.org/pdf/3459/345932037002.pdf 
Presas, M. (1997). La verdad de la ficción. Buenos Aires: Corregidor.

Randall, M. (1992). ¿Qué es, y cómo se hace un testimonio? Revista de Crítica Literaria Latinoamericana, XVIII(36), 21-45. Doi: 10.2307/4530621

Ricoeur, P. (1983). Tiempo y narración. Configuración del tiempo en el relato histórico. Madrid: Editorial Siglo XXI.

Ricoeur, P. (1999). La lectura del tiempo pasado: Memoria y olvido. España: Editorial Arrecife.

Santillán, G., Keller, M. y Cisterna, M. (2010). Sobreviviendo a un tsunami: Lecciones de Chile, Hawái y Japón. Valparaíso: Universidad Católica de Valparaíso. Recuperado de http://www.sociedadgeologica.cl/
wp-content/uploads/2011/05/Sobreviviendo-a-unTsu-Lecciones-Chile-Hawai-y-Japon.pdf

Schuster, R., Salcedo, D. y Valenzuela, L. (2002). Overview of catastrophic landslides of South America in the twentieth century. En S. Evans y J. DeGraff (eds.), Catastrophic landslides: effects, occurrence, and mechanisms (pp.1-34). Colorado: Geological Society of America Reviews in Engineering Geology.

Subercaseaux, B. (2004). Historia de las ideas y de la cultura en Chile. Tomo IV. Nacionalismo y cultura. Santiago: Editorial Universitaria.

Taylor, S. y Bogdan, R. (1990). Introducción a los métodos cualitativos de investigación. Barcelona: Paidós.

Para citar este artículo

Abarca González, C. y Huaiquimil Hermosilla, A. (2018). Experiencia de articulación del discurso de la memoria en un taller de relatos del terremoto y tsunami de 1960. Folios, 48. 39-55 